\title{
SOME PROPERTIES OF ALTERNATIVE ESTIMATES OF THE COBB- DOUGLAS PRODUCTION FUNCTION
}

\author{
BY J. KMENTA
}

\begin{abstract}
The purpose of this note is to demonstrate the equivalence of indirect least squares and Hoch's generalized method of estimating the production function parameters. It is further shown that these estimates are, under appropriate assumptions, maximum likelihood estimates, and their asymptotic properties are noted.
\end{abstract}

LET US CONSIDER a perfectly competitive industry in which firms produce a homogeneous product and employ two homogeneous, variable, and substitutable inputs. ${ }^{1}$ The production function (of the Cobb-Douglas type) and the two profit maximizing conditions are, for the $i$ th firm,

$$
\begin{aligned}
& x_{0 i}=k_{0}+a_{1} x_{1 i}+a_{2} x_{2 i}+v_{0 i}, \\
& x_{1 i}=k_{1}+x_{0 i}+v_{1 i}, \\
& x_{2 i}=k_{2}+x_{0 i}+v_{2 i},
\end{aligned}
$$

where $x_{0 i}, x_{1 i}$ and $x_{2 i}$ are logarithms of the quantities of output and of input 1 and input 2, and $v_{0 i}, v_{1 i}$ and $v_{2 i}$ are stochastic disturbances. Following Marschak and Andrews [4], we shall refer to $v_{0 i}$ as the "technical" disturbance, and to $v_{1 i}$ and $v_{2 i}$ as the "economic" disturbances. The problem is to estimate the parameters $a_{1}$ and $a_{2}$ from observations of outputs and inputs in a sample of firms.

One approach to the estimation problem was suggested by Hoch [1], who specified the bias of single equation least squares estimates in infinitely large samples. Consistent estimates of $a_{1}$ and $a_{2}$ are obtained by correcting the simple least squares estimates for the asymptotic bias. Hoch distinguishes between the case of no correlation between the disturbances, and the case where the economic disturbances are correlated with each other but not with the technical disturbance. Estimates are derived for the uncorrelated case, and a procedure is indicated for the derivation of "generalized" estimates applicable to the case of nonzero correlation between $v_{1}$ and $v_{2}{ }^{2}$ The formula for Hoch's generalized estimates is

$$
\begin{array}{r}
\tilde{a}_{r}=\hat{a}_{r}\left[1+\frac{\hat{S}_{00}\left(\hat{S}_{11}+\hat{S}_{22}-2 \hat{S}_{12}\right)}{\hat{S}_{11} \hat{S}_{22}-\hat{S}_{12}^{2}}\right]-\tilde{S}_{00}\left(\hat{S}_{q q}-\hat{S}_{12}\right) /\left(\hat{S}_{11} \hat{S}_{22}-\hat{S}_{12}^{2}\right), \\
r=1,2 ; q=1,2 ; r \neq q,
\end{array}
$$

where $\hat{a}_{r}$ is the simple least squares estimate of $a_{r}$, and $\tilde{S}_{00}, \hat{S}_{11}, \hat{S}_{12}$, and $\hat{S}_{22}$

\footnotetext{
${ }^{1}$ Only two inputs are being considered in order to avoid cumbersome notation, but the proofs can easily be extended to include the more general case.

${ }^{2}$ For a full description of Hoch's generalized estimates, see Kmenta and Joseph [3].
} 
represent estimates of the variances and covariances of the disturbances. These can be obtained as follows:

and

$$
\begin{aligned}
& \hat{S}_{r r}=C_{00}+C_{r r}-2 C_{0 r}, \quad r=1,2, \\
& \hat{S}_{12}=C_{00}+C_{12}-C_{01}-C_{02}, \\
& \tilde{S}_{00}=\hat{S}_{00}\left(\hat{S}_{11} \hat{S}_{22}-\hat{S}_{12}^{2}\right) /\left[\left(\hat{S}_{11} \hat{S}_{22}-\hat{S}_{12}^{2}\right)-\hat{S}_{00}\left(\hat{S}_{11}+\hat{S}_{22}-2 \hat{S}_{12}\right)\right],
\end{aligned}
$$

where $C_{00}$ is the sample variance of $x_{0} ; C_{r r}$ is the sample variance of $x_{r} ; C_{12}$ is the sample covariance of $x_{1}$ and $x_{2}$; and $C_{0 r}$ is the sample covariance of $x_{0}$ and $x_{r}$.

The method of indirect least squares ${ }^{3}$ is based on the fact that, according to equations (2) and (3), $\left(x_{1}-x_{0}\right)$ and $\left(x_{2}-x_{0}\right)$ are independent of $v_{0}$, providing $v_{0}$ is independent of $v_{1}$ and $v_{2}$. If this is the case, we may form a new equation

$$
x_{0 i}=b_{0}+b_{1}\left(x_{1 i}-x_{0 i}\right)+b_{2}\left(x_{2 i}-x_{0 i}\right)+e_{i}
$$

and obtain simple least squares estimates of $b_{1}$ and $b_{2}$ which are consistent. Equation (5) can be rewritten as

$$
\begin{aligned}
x_{0 i}=b_{0} /\left(1+b_{1}+b_{2}\right)+\left[b_{1} /\left(1+b_{1}\right.\right. & \left.\left.+b_{2}\right)\right] x_{1 i} \\
+ & {\left[b_{2} /\left(1+b_{1}+b_{2}\right)\right] x_{2 i}+e_{i} /\left(1+b_{1}+b_{2}\right) . }
\end{aligned}
$$

A comparison of (5a) with the production function (1) shows that $a_{1}$ and $a_{2}$ may be expressed in terms of $b_{1}$ and $b_{2}$. If we replace $b_{1}$ and $b_{2}$ by their least squares estimates $\hat{b}_{1}$ and $\hat{b}_{2}$, we obtain the indirect least squares estimates as given by

$$
\dot{a}_{r}=\hat{b}_{r} /\left(1+\hat{b}_{1}+\hat{b}_{2}\right), \quad r=1,2 .
$$

These estimates are also consistent.

We shall now prove the equivalence of indirect least squares and Hoch's generalized estimates. By substituting for $\tilde{S}_{00}$ from (4c) into (4), we can write the formula for Hoch's generalized estimates as

$$
\tilde{a}_{r}=\frac{\hat{a}_{r}\left(\hat{S}_{11} \hat{S}_{22}-\hat{S}_{12}^{2}\right)-\hat{S}_{00}\left(\hat{S}_{q q}-\hat{S}_{12}\right)}{\left(\hat{S}_{11} \hat{S}_{22}-\hat{S}_{12}^{2}\right)-\hat{S}_{00}\left(\hat{S}_{11}+\hat{S}_{22}-\hat{S}_{12}\right)}, \quad r=1,2 ; q=1,2 ; r \neq q .
$$

The indirect least squares estimates of equation (6) can be expressed in terms of sample moments. Let

$$
\begin{aligned}
& x_{0 i}=z_{0 i}, \\
& x_{r i}-x_{0 i}=z_{r i}, \quad r=1,2,
\end{aligned}
$$

${ }^{3}$ For a description of this method, which was suggested by $\mathrm{H}$. Theil, see Hoch $[1$, p. 572, footnote 11] or Walters [6, p. 132]. Both references contain an initial error of sign which makes the final formulas incorrect. 
and

$$
M_{p q}=\frac{1}{n} \sum_{i=1}^{n}\left(z_{p i}-\bar{z}_{p}\right)\left(z_{q i}-\bar{z}_{q}\right), \quad p, q=0,1,2
$$

Then

$$
\hat{b}_{r}=\left(M_{q q} M_{0 r}-M_{12} M_{0 q}\right) /\left(M_{11} M_{22}-M_{12}^{2}\right),
$$

and

$$
\dot{a}_{r}=\left(M_{q q} M_{0 r}-M_{12} M_{0 q}\right) / A, \quad r=1,2 ; q=1,2 ; r \neq q,
$$

where $A=M_{11} M_{22}-M_{12}^{2}+M_{22} M_{01}+M_{11} M_{02}-M_{12} M_{01}-M_{12} M_{02}$. Sample moments referring to the $z$ 's can readily be expressed in terms of sample moments referring to the $x$ 's. In fact, we have

$$
\begin{aligned}
& M_{00}=C_{00}, \\
& M_{0 r}=C_{0 r}-C_{00}, \\
& M_{r r}=C_{r r}-2 C_{0 r}+C_{00}, \\
& M_{12}=C_{12}-C_{01}-C_{02}+C_{00}, \quad r=1,2 .
\end{aligned}
$$

By substitution into (8) we obtain

$$
\begin{array}{r}
\dot{a}_{r}=\left[C_{q q}\left(C_{0 r}-C_{00}\right)-C_{12}\left(C_{0 q}-C_{00}\right)-C_{0 q}\left(C_{01}-C_{02}\right)\right] / B, \\
r=1,2 ; q=1,2 ; r \neq q,
\end{array}
$$

where $B=C_{22}\left(C_{11}-C_{01}\right)+C_{02}\left(C_{12}-C_{11}\right)+C_{12}\left(C_{01}-C_{12}\right)$.

Introducing Hoch's notation of (4a) and (4b) we get

$$
\dot{a}_{r}=\left[\hat{S}_{q q}\left(C_{0 r}-C_{00}\right)-\hat{S}_{12}\left(C_{0 q}-C_{00}\right)\right] / E, \quad r=1,2 ; q=1,2 ; r \neq q,
$$

where $E=\hat{S}_{11} \hat{S}_{22}-\hat{S}_{12}^{2}+\hat{S}\left({ }_{11} C_{02}-C_{00}\right)+\hat{S}_{22}\left(C_{01}-C_{00}\right)-\hat{S}_{12}\left(C_{01}+C_{02}-2 C_{00}\right)$. Further, the simple least squares estimate $\hat{a}_{r}$ is given by the "normal" equation

$$
C_{0 r}=\hat{a}_{r} C_{r r}+\hat{a}_{q} C_{12}, \quad r=1,2 ; q=1,2 ; r \neq q .
$$

Substituting into the above from (4a), (4b), and (4d) leads to

$$
\begin{aligned}
& C_{01}-C_{00}=\left(\hat{a}_{1} \hat{S}_{11}+\hat{a}_{2} \hat{S}_{12}-\hat{S}_{00}\right) /\left(1-\hat{a}_{1}-\hat{a}_{2}\right), \\
& C_{02}-C_{00}=\left(\hat{a}_{1} \hat{S}_{12}+\hat{a}_{2} \hat{S}_{22}-\hat{S}_{00}\right) /\left(1-\hat{a}_{1}-\hat{a}_{2}\right) .
\end{aligned}
$$

Consequently, (10) can be written as

$$
\dot{a}_{r}=\left[\hat{a}_{r}\left(\hat{S}_{11} \hat{S}_{22}-\hat{S}_{12}^{2}\right)-\hat{S}_{00}\left(\hat{S}_{q q}-\hat{S}_{12}\right)\right] / F, \quad r=1,2 ; q=1,2 ; r \neq q,
$$

where $F=\left(\hat{S}_{11} \hat{S}_{22}-\hat{S}_{12}^{2}\right)-\hat{S}_{00}\left(\hat{S}_{11}+\hat{S}_{22}-2 \hat{S}_{12}\right)$. 
Obviously, (13) is the same as (7). This proves that Hoch's generalized estimates are identically equivalent to the indirect least squares estimates. ${ }^{4}$

If it is assumed that the disturbances of the system described by equations (1) to (3) are normally distributed, and that the technical disturbance $v_{0}$ is independent of the economic disturbances $v_{1}$ and $v_{2}$, we can form the following likelihood function:

$$
\begin{aligned}
L= & -\frac{3 n}{2} \log (2 \pi)+n \log \operatorname{det} J-\frac{n}{2} \log \operatorname{det} \Sigma \\
& -\sum_{i=1}^{n}\left(x_{0 i}^{\prime}-a_{1} x_{1 i}^{\prime}-a_{2} x_{2 i}^{\prime}\right)^{2} / 2 S_{00} \\
& -S_{22} \sum_{i=1}^{n}\left(x_{1 i}^{\prime}-x_{0 i}^{\prime}\right)^{2} / 2\left(S_{11} S_{22}-S_{12}^{2}\right) \\
& -S_{11} \sum_{i=1}^{n}\left(x_{2 i}^{\prime}-x_{0 i}^{\prime}\right)^{2} / 2\left(S_{11} S_{22}-S_{12}^{2}\right) \\
& +S_{12} \sum_{i=1}^{n}\left(x_{1 i}^{\prime}-x_{0 i}^{\prime}\right)\left(x_{2 i}^{\prime}-x_{0 i}^{\prime}\right) /\left(S_{11} S_{22}-S_{12}^{2}\right), \\
\text { where } \quad J= & {\left[\begin{array}{ccc}
+1 & -a_{1} & -a_{2} \\
-1 & +1 & 0 \\
-1 & 0 & +1
\end{array}\right], }
\end{aligned}
$$

and $\Sigma$ is the variance-covariance matrix of the disturbances given by

$$
\Sigma=\left[\begin{array}{lll}
S_{00} & 0 & 0 \\
0 & S_{11} & S_{12} \\
0 & S_{12} & S_{22}
\end{array}\right] .
$$

The variables $x_{0 i}^{\prime}, x_{1 i}^{\prime}$ and $x_{2 i}^{\prime}$ are the observable logarithmic outputs and inputs measured in terms of deviations from their respective sample means. The maximum likelihood estimates of the parameters thus obtained are exactly the same as those obtained by Hoch's generalized method or by the indirect least squares method. ${ }^{5}$

It is also interesting to note that the so-called "moments" method of estimating the production function parameters suggested by Marschak and Andrews in [4] leads to the same estimates, providing only that the assumption $E\left(v_{0} v_{1}\right)=E\left(v_{0} v_{2}\right)=0$ is made. ${ }^{6}$ Equating the variances and covariances of the variables in equations (1) to (3) gives

$$
J X J^{\prime}=\Sigma,
$$

${ }^{4}$ In the Monte Carlo experiment presented in [3], indirect least squares and Hoch's generalized estimates were obtained in some of the models. The numerical discrepancies shown were very small and can be accounted for by errors due to rounding.

5 The solution is, of course, subject to the condition that $a_{1}+a_{2}<1$.

${ }^{6}$ This assumption was, in fact, not made by Marschak and Andrews. Also, the model of Marschak and Andrews was a more general one since it allowed for imperfect competition. 
where $X$ is a matrix of the variances and covariances of the $x$ 's, and $J$ and $\Sigma$ are defined as before. By substituting sample values into $X$ we have, because of symmetry, six equation to estimate $a_{1}, a_{2}$, and the four variances and covariances of the disturbances. These estimates are the same as the maximum likelihood estimates above. $^{7}$

The maximum likelihood property of Hoch's generalized, indirect least squares, and moments estimates enables us to derive their asymptotic variances by determining the diagonal elements of the matrix ${ }^{8}$

$$
\left\|-\frac{\partial^{2} L}{\partial \theta_{i} \partial \theta_{j}} \mid \begin{array}{c}
\theta_{1}=\tilde{\theta}_{1} \\
\dot{\theta_{k}=\tilde{\theta}_{k}}
\end{array}\right\|^{-1},
$$

where the $\theta$ 's represent the parameters and the $\tilde{\theta}$ 's their maximum likelihood estimates. An inspection of the likelihood function shows that the matrix of the second derivatives is quasi-diagonal. Substituting second partial derivatives of the likelihood function at the points given by the maximum likelihood estimates gives the following results: ${ }^{9}$

$$
\operatorname{plim} \operatorname{Var}\left(\tilde{a}_{1}\right)=\operatorname{plim} \operatorname{Var}\left(\tilde{a}_{2}\right)=\tilde{S}_{00} / n\left(\hat{S}_{11}+\hat{S}_{22}-2 \hat{S}_{12}\right)
$$

For the purpose of computation, equation (15) can be rewritten as

$$
\begin{aligned}
& \operatorname{plim} \operatorname{Var}\left(\tilde{a}_{1}\right)=\operatorname{plim} \operatorname{Var}\left(\tilde{a}_{2}\right)=\left(1-\tilde{a}_{1}-\tilde{a}_{2}\right)\left(C_{00}-\tilde{a}_{1} C_{01}-\tilde{a}_{2} C_{02}\right) / \\
& n\left(C_{11}+C_{22}-2 C_{12}\right) .
\end{aligned}
$$

A comparison with the numerical results obtained in the Monte Carlo experiment in [3] indicates that indirect least squares estimates from samples of size 100 have a dispersion which is reasonably closely approximated by (15).

A necessary condition for the consistency of the maximum likelihood estimates is that $E\left(v_{0} v_{1}\right)=S_{01}=0$, and $E\left(v_{0} v_{2}\right)=S_{02}=0 .{ }^{10}$ If the assumption that $S_{01}=S_{02}=$ 0 is not fulfilled, it may be interesting to inquire about the degree of inconsistency involved. Let us consider the maximum likelihood estimates as determined by the indirect least squares formula (8). We may write

7 The equivalence of moments and indirect least squares (and instrumental variables) estimates was also derived by Yair Mundlak in [5, section 4.3]. The author is indebted to the referees for drawing his attention to Mundlak's study.

8 See, e.g., Klein [2, p. 141].

9 The solution is subject to the condition that $\tilde{a}_{1}+\tilde{a}_{2} \neq 1$.

10 The assumption that $S_{12}=0$ is only "optional" and in any case does not alter the estimation formula. However, a high degree of relationship between $v_{1}$ and $v_{2}$ is likely to lead to multicollinearity problems. 


$$
\begin{aligned}
\operatorname{plim} M_{r r} & =S_{r r}, \\
\operatorname{plim} M_{12} & =S_{12}, \\
\operatorname{plim} M_{0 r} & =\left(a_{r} S_{r r}+a_{q} S_{12}+S_{0 r}\right) /\left(1-a_{1}-a_{2}\right), \\
\operatorname{plim} M_{00} & =\left(a_{1}^{2} S_{11}+a_{2}^{2} S_{22}+S_{00}+2 a_{1} a_{2} S_{12}+2 a_{1} S_{01}+2 a_{2} S_{02}\right) / \\
& \left(1-a_{1}-a_{2}\right), \quad r=1,2 ; q=1,2 ; r \neq q .
\end{aligned}
$$

Substitution into (8) gives

$$
\operatorname{plim} \dot{a}_{r}=\left[a_{r}\left(S_{11} S_{22}-S_{12}^{2}\right)+S_{q q} S_{0 r}-S_{12} S_{0 q}\right] / G,
$$

where $G=S_{11} S_{22}-S_{12}^{2}+S_{11} S_{02}+S_{22} S_{01}-S_{12} S_{01}-S_{12} S_{02}$. The coefficient of correlation between $v_{0}$ and $v_{r}(r=1,2)$ is

$$
R_{0 r}=S_{0 r} / \sqrt{S_{00} S_{r r}}, \quad r=1,2,
$$

and (17) may be rewritten as

$$
\operatorname{plim} \dot{a}_{r}=\left[a_{r} S_{11} S_{22}\left(1-R_{12}^{2}\right)+S_{q q} \sqrt{S_{00} S_{r r}}\left(R_{0 r}-R_{12} R_{0 q}\right)\right] / H
$$

where $H=S_{11} S_{22}\left(1-R_{12}^{2}\right)+S_{q q} \sqrt{S_{00} S_{r r}}\left(R_{0 r}-R_{12} R_{0 q}\right), r=1,2 ; q=1,2 ; r \neq q$. Equation (18) enables us to find the degree of inconsistency in cases in which the values of the correlation coefficients are known or can be assumed.

\section{University of Wisconsin}

\section{REFERENCES}

[1] Hoch, IRving: "Simultaneous Equation Bias in the Context of the Cobb-Douglas Production Function," Econometrica, 26 (October, 1958), 566-578.

[2] Klein, L. R.: A Textbook of Econometrics, New York: Row, Peterson, and Co., 1953.

[3] KMENTA, J., AND M. E. JosePh: "A Monte Carlo Study of Alternative Estimates of the CobbDouglas Production Function," Econometrica, 31 (July, 1963), 363-385.

[4] MarschaK, J., AND W. H. ANdrews: "Random Simultaneous Equations and the Theory of Production," Econometrica, 12 (July-October, 1944), 143-205.

[5] Mundlak, YaIR: "Estimation of Production and Behavioral Functions from a Combination of Cross-Section and Time Series Data," revised version of a paper that was read and distributed at the meeting of the Econometric Society, New York, December, 1961 ; to be published in D. Patinkin (ed.): Measurement in Economics-Essays in Memory of Yehuda Grunfeld (Stanford: Stanford University Press; forthcoming in 1963).

[6] Walters, A. A.: "Some Notes on the Cobb-Douglas Production Function," Metroeconomica, 13 (December, 1961), 121-138. 